\title{
Stochastic Stability and Stabilization
}

\author{
Xuerong Mao \\ Department of Statistics and Modelling Science \\ University of Strathclyde \\ Glasgow G1 1XH, U.K.
}

e-mail: xuerong@stams.strath.ac.uk

Tel: $+44-141-5483669$

Fax: $+44-141-5522079$ 


\section{Introduction}

- Stochastic modelling

- Automatic control of stochastic systems

- Analysis of stability in stochastic models

- Itô's stochastic calculus

- Lyapunov's second method

- Aims of this talks:

The original ideas of stochastic stability have been refined, generalized and extended in several directions and many new concepts have been introduced. Especially, some new ideas and approaches which might provide an exciting prospect of further advancement are still in the initial stages of investigation. In this talk, some of these aspects will be discussed. 


\section{Notations}

- $\left(\Omega, \mathcal{F},\left\{\mathcal{F}_{t}\right\}_{t \geq 0}, P\right)$ : a complete probability space.

- $B(t)=\left(B_{1}(t), \cdots, B_{m}(t)\right)^{T}$ : an $m$-dimensional Brownian motion.

- $f: R^{n} \times R_{+} \rightarrow R^{n}$ and $g: R^{n} \times R_{+} \rightarrow R^{n \times m}$ satisfy the local Lipschitz condition and the linear growth condition.

- The $n$-dimensional stochastic differential equation (SDE)

$$
d x(t)=f(x(t), t) d t+g(x(t), t) d B(t), \quad t \geq 0, x(0)=x_{0} \in R^{n} .
$$

The unique continuous solution is denoted by $x\left(t ; x_{0}\right)$.

- $C^{2,1}\left(R^{n} \times R_{+} ; R_{+}\right)$: the family of all nonnegative functions $V(x, t)$ on $R^{n} \times R_{+}$which are twice continuously differentiable in $x$ and once in $t$.

- The diffusion operator $L$ acting on $C^{2,1}\left(R^{n} \times R_{+} ; R_{+}\right)$functions is defined by

$$
\begin{aligned}
& L V(x, t)=V_{t}(x, t)+V_{x}(x, t) f(x, t)+\frac{1}{2} \operatorname{trace}\left[g^{T}(x, t) V_{x x}(x, t) g(x, t)\right], \\
& \text { where } V_{x}=\left(V_{x_{1}}, \cdots, V_{x_{n}}\right) \text { and } V_{x x}=\left(V_{x_{i} x_{j}}\right)_{n \times n} .
\end{aligned}
$$

- $\mathcal{K}$ : the class of continuous (strictly) increasing functions $\mu$ from $R_{+}$to $R_{+}$with $\mu(0)=0$. 
- $\mathcal{K}_{\infty}$ : the class of functions $\mu$ in $\mathcal{K}$ with $\mu(r) \rightarrow \infty$ as $r \rightarrow \infty$.

- $L^{1}\left(R_{+} ; R_{+}\right)$: the family of all functions $\gamma: R_{+} \rightarrow R_{+}$such that $\int_{0}^{\infty} \gamma(t) d t<\infty$.

- $|\cdot|$ : the Euclidean norm for vectors or the trace norm of matrices.

- $A^{T}$ : the transpose of a vector or matrix $A$. 
3 A Classical Result and Question

Theorem 3.1 Assume that there are functions $V \in C^{2,1}\left(R^{n} \times\right.$ $\left.R_{+} ; R_{+}\right), \mu_{1}, \mu_{2} \in \mathcal{K}_{\infty}$ and $\mu_{3} \in \mathcal{K}$ such that

$$
\mu_{1}(|x|) \leq V(x, t) \leq \mu_{2}(|x|)
$$

and

$$
L V(x, t) \leq-\mu_{3}(|x|)
$$

for all $(x, t) \in R^{n} \times R_{+}$. Then, for every $x_{0} \in R^{n}$,

$$
\lim _{t \rightarrow \infty}\left|x\left(t ; x_{0}\right)\right|=0 \quad \text { a.s. }
$$

Question: Do we still have asymptotic stability if $V$ is not positive-definite or $L V$ is not negative-definite?

This is not possible if the underlying system is an ordinary differential equation (ODE) but possible if it is an SDE. 


\section{Stochastic Stability}

The theorems discussed below not only describe this situation but also show that stability theory for SDEs is much richer than that for ODEs.

Theorem 4.1 (Mao [15]) Assume that there are functions $V \in$ $C^{2,1}\left(R^{n} \times R_{+} ; R_{+}\right), \mu_{1}, \mu_{2}, \mu_{3} \in \mathcal{K}$ and $\gamma \in L^{1}\left(R_{+} ; R_{+}\right)$such that (3.1) is satisfied while (3.2) is replaced by the following weaker condition

$L V(x, t) \leq \gamma(t) \wedge\left[\gamma(t)+\left|V_{x}(x, t) g(x, t)\right|^{2}-\mu_{3}(|x|)\right], \quad \forall(x, t) \in R^{n} \times R_{+}$.

Then, for every $x_{0} \in R^{n}$,

$$
\lim _{t \rightarrow \infty}\left|x\left(t ; x_{0}\right)\right|=0 \quad \text { a.s. }
$$


Example 4.2 Consider a 2-dimensional equation

$$
d\left[\begin{array}{l}
x_{1}(t) \\
x_{2}(t)
\end{array}\right]=\left[\begin{array}{c}
-x_{1}(t)+x_{1}(t) x_{2}(t)+2 e^{-t} \\
-x_{1}^{2}(t)-2 x_{2}(t)
\end{array}\right] d t+\left[\begin{array}{c}
e^{-t} d B_{1}(t) \\
2 x_{2}(t) d B_{2}(t)
\end{array}\right],
$$

where $\left(B_{1}(t), B_{2}(t)\right)$ is a two-dimensional Brownian motion. Let $V(x)=$ $|x|^{2}$ and compute

$$
\begin{aligned}
L V(x, t) & =2 x_{1}\left(-x_{1}+x_{1} x_{2}+2 e^{-t}\right)+2 x_{2}\left(-x_{1}^{2}-2 x_{2}\right)+e^{-2 t}+4 x_{2}^{2} \\
& =e^{-2 t}+4 x_{1} e^{-t}-2 x_{1}^{2} \leq 5 e^{-2 t}-x_{1}^{2} \\
& \leq 5 e^{-2 t} .
\end{aligned}
$$

Clearly, $L V$ is not negative definite so we can not apply the classical Theorem 3.1 using the Lyapunov function above to deduce the asymptotic stability in probability 1 . However, we can apply Theorem 4.1. In fact, compute

$$
\left|V_{x}(x, t) g(x, t)\right|^{2}=4 x_{1}^{2} e^{-2 t}+16 x_{2}^{4}
$$

SO

$$
5 e^{-2 t}-L V(x, t)+\left|V_{x}(x, t) g(x, t)\right|^{2} \geq x_{1}^{2}+16 x_{2}^{4} \geq \mu_{3}(|x|),
$$

where

$$
\mu_{3}(r)=\min _{|x| \geq r}\left[x_{1}^{2}+16 x_{2}^{4}\right] \quad \text { for } r \geq 0,
$$

which is in $\mathcal{K}$ clearly. Thus

$$
L V(x, t) \leq 5 e^{-2 t}+\left|V_{x}(x, t) g(x, t)\right|^{2}-\mu_{3}(|x|) .
$$


We can therefore conclude by Theorem 4.1 that the solution of equation (4.2) starting from any where in $R^{2}$ will tend to zero asymptotically with probability one.

Theorem 4.3 (Mao [13]) Assume that there is a function $V \in$ $C^{2,1}\left(R^{d} \times R_{+} ; R_{+}\right)$and constants $p>0, c_{1}>0, c_{2} \in R, c_{3} \geq 0$, such that

$$
c_{1}|x|^{p} \leq V(x, t), \quad L V(x, t) \leq c_{2} V(x, t)
$$

and

$$
\left|V_{x}(x, t) g(x, t)\right|^{2} \geq c_{3} V^{2}(x, t) .
$$

Then

$$
\limsup _{t \rightarrow \infty} \frac{1}{t} \log \left|x\left(t ; x_{0}\right)\right| \leq-\frac{c_{3}-2 c_{2}}{2 p} \quad \text { a.s. }
$$

for all $x_{0} \in R^{n}$. In particular, if $c_{3}>2 c_{2}$, the solution of equation (2.1) will tend to zero exponentially with probability one. 
In the above theorems we still require $V$ to be positivedefinite. What happens if it is not?

Theorem 4.4 Assume that there are $V \in C^{2,1}\left(R^{n} \times R_{+} ; R_{+}\right)$, $\gamma \in L^{1}\left(R_{+} ; R_{+}\right)$and $w \in C\left(R^{n} ; R_{+}\right)$such that

$$
\lim _{|x| \rightarrow \infty} \inf _{0 \leq t<\infty} V(x, t)=\infty
$$

and

$$
L V(x, t) \leq \gamma(t)-w(x), \quad(x, t) \in R^{n} \times R_{+} .
$$

Assume also that for any $h>0$ there is a $K_{h}>0$ such that

$$
|f(x, t)| \vee|g(x, t)| \leq K_{h}
$$

for all $t \geq 0$ and $x \in R^{n}$ with $|x| \leq h$. Then,

$$
\operatorname{Ker}(w):=\left\{x \in R^{n}: w(x)=0\right\} \neq \emptyset .
$$

Moreover, the solution of equation (2.1) has the properties that the limit $\lim _{t \rightarrow \infty} V\left(x\left(t ; x_{0}\right), t\right)$ exists and is finite almost surely, and

$$
\lim _{t \rightarrow \infty} d\left(x\left(t ; x_{0}\right), \operatorname{Ker}(w)\right)=0 \quad \text { a.s. }
$$

where $d(x, A)$ denotes the Haussdorf semi-distance between $x \in$ $R^{n}$ and the set $A \subset R^{n}$, that is, $d(x, A)=\inf _{y \in A}|x-y|$. 
It follows from this theorem that if, in addition, $w(x)=0$ iff $x=0$, namely $\operatorname{Ker}(w)=\{0\}$, then

$$
\lim _{t \rightarrow \infty} x\left(t ; x_{0}\right)=0 \quad \text { a.s. }
$$

In other words, we still have asymptotic stability although $V$ may not be positive-definite. 
Most of the existing results on stochastic stability use a single Lyapunov function, but the recent new trend is to use multiple Lyapunov functions.

Theorem 4.5 Assume that there are functions $V, U \in C^{2,1}\left(R^{n} \times\right.$ $\left.R_{+} ; R_{+}\right), \gamma_{1}, \gamma_{2} \in L^{1}\left(R_{+} ; R_{+}\right)$as well as $\rho \in C\left(R_{+} ; R_{+}\right)$, such that

$$
L V(x, t) \leq \gamma_{1}(t), \quad L U(x, t) \leq \gamma_{2}(t)
$$

and

$$
\gamma_{1}(t)-L V(x, t)+\left|V_{x}(x, t) g(x, t)\right|^{2} \geq \rho(U(x, t))
$$

for all $(x, t) \in R^{n} \times R_{+}$. Then $D_{\rho}=\{u \geq 0: \rho(u)=0\} \neq \emptyset$, and

$$
\lim _{t \rightarrow \infty} U\left(x\left(t ; x_{0}\right), t\right) \in D_{\rho} \quad \text { a.s. }
$$

for every $x_{0} \in R^{n}$. 
Theorem 4.6 Let all the assumptions of Theorem 4.5 hold.

(i) If $D_{\rho}$ is bounded and

$$
\liminf _{|x| \rightarrow \infty, t \rightarrow \infty} U(x, t)>\max \left\{u: u \in D_{\rho}\right\},
$$

then there is a constant $K>0$ such that for every $x_{0} \in R^{n}$,

$$
\limsup _{t \rightarrow \infty}\left|x\left(t ; x_{0}\right)\right| \leq K \quad \text { a.s. }
$$

(ii) If $D_{\rho}=\{0\}$ and for some $\mu \in \mathcal{K}$,

$$
\mu(|x|) \leq U(x, t) \quad \forall(x, t) \in R^{n} \times R_{+},
$$

then for every $x_{0} \in R^{n}$,

$$
\lim _{t \rightarrow \infty}\left|x\left(t ; x_{0}\right)\right|=0 \quad \text { a.s. }
$$




\section{Stochastic Stabilization}

It is not surprising that noise can destablize a stable system, but do you believe that noise can also stabilise an unstable system?

Consider a scalar unstable system

$$
\dot{y}(t)=y(t) \quad \text { on } t \geq 0, y(0)=x_{0} \in R .
$$

Perturb this system by noise and suppose that the perturbed system has the form

$$
d x(t)=x(t) d t+2 x(t) d B(t) \quad \text { on } t \geq 0, x(0)=x_{0} \in R,
$$

where $B(t)$ is a one-dimensional Brownian motion. This SDE has the explicit solution

$$
\left.x(t)=x_{0} \exp [-t+2 B(t))\right],
$$

which yields immediately that

$$
\lim _{t \rightarrow \infty} \frac{1}{t} \log |x(t)|=-1 \quad \text { a.s. }
$$

That is, the SDE becomes stable. In other words, the noise has stabilized the unstable system. 


\section{A brief history:}

- Has'minskii (1969): The pioneering work where two white noise sources were used to stabilize a particular system.

- Arnold, Crauel \& Wihstutz (1983) and Arnold (1990): Any linear system $\dot{x}(t)=A x(t)$ with trace $(A)<0$ can be stabilized by one real noise source.

- Scheutzow (1993): Stochastic stabilization for two special nonlinear systems.

- Mao (1994): The general theory on stochastic stabilization for nonlinear SDEs.

- Mao (1996): Design a stochastic control that can self-stabilize the underlying system.

- Caraballo, Liu and Mao (2001): Stochastic stabilization for partial differential equations (PDEs). 


\section{Stochastic stabilization}

Given a nonlinear ODE

$$
\dot{y}(t)=f(y(t), t) \quad \text { on } t \geq 0, y(0)=x_{0} \in R^{n}
$$

we use the $m$-dimensional Brownian motion $B(t)$ as the source of noise to perturb it. Suppose the stochastically perturbed system is described by the semilinear SDE

$$
d x(t)=f(x(t), t) d t+\sum_{i=1}^{m} G_{i} x(t) d B_{i}(t)
$$

on $t \geq 0, x(0)=x_{0} \in R^{n}$, where $G_{i}, 1 \leq i \leq m$, are all $n \times n$ matrices.

\section{Condition:}

Assume that $f: R^{n} \times R_{+} \rightarrow R^{n}$ is a locally Lipschitz continuous function and particularly, for some $K>0$,

$$
|f(x, t)| \leq K|x| \quad \text { for all }(x, t) \in R^{n} \times R_{+} \text {. }
$$


Theorem 5.1 Let (5.3) hold. Assume that there are two constants $\lambda>0$ and $\rho \geq 0$ such that

$$
\sum_{i=1}^{m}\left|G_{i} x\right|^{2} \leq \lambda|x|^{2} \quad \text { and } \quad \sum_{i=1}^{m}\left|x^{T} G_{i} x\right|^{2} \geq \rho|x|^{4}
$$

for all $x \in R^{n}$. Then

$$
\limsup _{t \rightarrow \infty} \frac{1}{t} \log \left|x\left(t ; x_{0}\right)\right| \leq-\left(\rho-K-\frac{\lambda}{2}\right) \quad \text { a.s. }
$$

for all $x_{0} \in R^{n}$. In particular, if $\rho>K+\frac{1}{2} \lambda$, then the noise stabilizes the given ODE (5.1).

Theorem 5.1 ensures that there are many choices for the matrices $G_{i}$ in order to stabilize a given system. For illustration, choose positivedefinite matrices $D_{i}$ such that

$$
x^{T} D_{i} x \geq \frac{\sqrt{3}}{2}\left\|D_{i}\right\||x|^{2}, \quad 1 \leq i \leq m .
$$

Obviously, there are many such matrices. Let $\sigma$ be a constant and $G_{i}=\sigma D_{i}$. Then

$$
\sum_{i=1}^{m}\left|G_{i} x\right|^{2} \leq \sigma^{2} \sum_{i=1}^{m}\left\|D_{i}\right\|^{2}|x|^{2}
$$

and

$$
\sum_{i=1}^{m}\left|x^{T} G_{i} x\right|^{2} \geq \frac{3 \sigma^{2}}{4} \sum_{i=1}^{m}\left\|D_{i}\right\|^{2}|x|^{4} .
$$

By Theorem 5.1, the solution of the SDE (5.2) satisfies

$$
\limsup _{t \rightarrow \infty} \frac{1}{t} \log \left|x\left(t ; x_{0}\right)\right| \leq-\left(\frac{\sigma^{2}}{4} \sum_{i=1}^{m}\left\|D_{i}\right\|^{2}-K\right) \quad \text { a.s. }
$$


Therefore the noise stabilizes the given ODE (5.1) if

$$
\sigma^{2}>\frac{4 K}{\sum_{i=1}^{m}\left\|D_{i}\right\|^{2}} .
$$

To conclude we state:

Theorem 5.2 Any nonlinear system $\dot{y}(t)=f(y(t), t)$ can be stabilized by Brownian motions provided (5.3) is satisfied. 


\section{Stochastic self-stabilization}

Consider an Itô equation with parameter $u>0$ of the form

$$
d x(t)=f(x(t), t) d t+u g(x(t), t) d B(t) .
$$

Assume that for all sufficiently large $u$, equation (5.6) is almost surely $L^{1}\left(R_{+} ; R^{n}\right)$-stable in the sense

$$
\int_{0}^{\infty}|x(t)| d t<\infty \quad \text { a.s. }
$$

Now replace $u$ by a control law

$$
u=\int_{0}^{t}|x(s)| d s
$$

and then equation (5.6) becomes a controlled system

$$
d x(t)=f(x(t), t) d t+\left[\int_{0}^{t}|x(s)| d s\right] g(x(t), t) d B(t) .
$$

It is "reasonable" to hope that this controlled system (5.8) is almost surely $L^{1}\left(R_{+} ; R^{n}\right)$-stable. In other words, equation (5.6) can be self-stabilized by the control law (1.2). 


\section{A "reasonable" argument}

The "reason" for this hope is as follows: If system (5.8) is not almost surely $L^{1}\left(R_{+} ; R^{n}\right)$-stable, then for some $\omega \in \Omega$ (with positive probability) $\int_{0}^{\infty}|x(t, \omega)| d t=\infty$. Consequently for all large $t$, $\int_{0}^{t}|x(s, \omega)| d s$ will be sufficiently large. Therefore, by the property of equation (5.6), it seems $\int_{0}^{\infty}|x(t, \omega)| d t<\infty$ but this is a contradiction. So system (5.8) should be almost surely $L^{1}\left(R_{+} ; R^{n}\right)$ stable.

Assumption 5.3 There is a symmetric positive-definite $n \times n$ matrix $Q$ and three positive constants $K, \alpha, \beta$ with $2 \beta>\alpha$ such that

$$
\begin{aligned}
\left|x^{T} Q f(x, t)\right| & \leq K|x|^{2}, \\
\operatorname{trace}\left(g^{T}(x, t) Q g(x, t)\right) & \leq \alpha x^{T} Q x, \\
\left|x^{T} Q g(x, t)\right|^{2} & \geq \beta\left|x^{T} Q x\right|^{2}
\end{aligned}
$$

for all $(x, t) \in R^{n} \times R_{+}$. 
Theorem 5.4 Under Assumption 5.3, the controlled system (5.8) is almost surely $L^{1}\left(R_{+} ; R^{n}\right)$-stable. More precisely, the solution of (5.8) has the property that either

$$
\int_{0}^{\infty}\left|x\left(t ; x_{0}\right)\right| d t \leq \sqrt{\frac{2 K}{(2 \beta-\alpha) \lambda_{\min }(Q)}}
$$

or

$$
\limsup _{t \rightarrow \infty} \frac{1}{t} \log \left(\left|x\left(t ; x_{0}\right)\right|\right)<0
$$

holds for almost all $\omega \in \Omega$. 
A new control law

$$
u=\int_{0}^{t}|r(s) x(s)|^{p} d s,
$$

where $p>0$ and $r(\cdot)$ is a continuous $R^{d \times n}$-valued function defined on $R_{+}$, which is called a convergence rate function.

The controlled system

$$
d x(t)=f(x(t), t) d t+\left[\int_{0}^{t}|r(s) x(s)|^{p} d s\right] g(x(t), t) d B(t)
$$

on $t \geq 0$ with initial value $x(0)=x_{0} \in R^{n}$.

- If we want to control the $i$-th component of the solution with the property

$$
\int_{0}^{\infty}\left|x_{i}\left(t ; x_{o}\right)\right|^{p} d t<\infty \quad \text { a.s. }
$$

we can choose the convergence rate function

$$
r(t)=(\underbrace{0, \cdots, 0,1}_{i \text { times }}, 0, \cdots, 0)_{1 \times n} .
$$

- If we want to control the solution with the property

$$
\int_{0}^{\infty} e^{\gamma t}\left|x\left(t ; x_{o}\right)\right|^{p} d t<\infty \quad \text { a.s. }
$$

we can choose $r(t)=e^{\gamma t / p} I_{n \times n}$, where $I_{n \times n}$ is the $n \times n$ identity matrix. 
Assumption 5.5 There exists a pair of positive constants $M$ and $\gamma$ such that

$$
|r(t)| \leq M e^{\gamma t} \quad \forall t \geq 0 .
$$

Theorem 5.6 Under Assumptions 5.3 and 5.5, the solution of the controlled system (5.10) has the property that

$$
\int_{0}^{\infty}\left|r(t) x\left(t ; x_{0}\right)\right|^{p} d t<\infty \quad \text { a.s. }
$$

How to stabilize equation (5.6) in order to have almost surely asymptotic stability or exponential stability? 
Control law

$$
u=\sup _{0 \leq s \leq t}|r(s) x(s)|
$$

Controlled system

$$
d x(t)=f(x(t), t) d t+\left[\sup _{0 \leq s \leq t}|r(s) x(s)|\right] g(x(t), t) d B(t)
$$

on $t \geq 0$ with initial data $x(0)=x_{0} \in R^{n}$.

Theorem 5.7 Under Assumptions 5.3 and 5.5, the solution of the controlled system (5.12) satisfies

$$
\sup _{0 \leq t<\infty}\left|r(t) x\left(t ; x_{0}\right)\right|<\infty \quad \text { a.s. }
$$

Furthermore,

(i) if $\lambda_{\min }\left(r^{T}(t) r(t)\right) \rightarrow \infty$ as $t \rightarrow \infty$, then

$$
\lim _{t \rightarrow \infty}\left|x\left(t ; x_{0}\right)\right|=0 \quad \text { a.s. }
$$

(ii) if

$$
\liminf _{t \rightarrow \infty} \frac{1}{t} \log \left[\lambda_{\min }\left(r^{T}(t) r(t)\right)\right] \geq \lambda
$$

for some positive constant $\lambda$, then

$$
\limsup _{t \rightarrow \infty} \frac{1}{t} \log \left(\left|x\left(t ; x_{0}\right)\right|\right) \leq-\frac{\lambda}{2} \quad \text { a.s. }
$$




\section{Conclusions}

- Traditionally speaking, one requires the Lyapunov function $V$ to be positive definite and $L V$ negative definite in order to have stochastic asymptotic stability.

- The recent research shows that we still have stochastic asymptotic stability even if we lose these positive and negative definiteness.

- This is due to the fact the noise has an effect of stabilization.

- Making use of this nice effect we show a number of theorems on stochastic asymptotic stability where the Lyapunov function $V$ may not be positive definite nor $L V$ negative definite.

- Making use of the stabilization effect of noise we design different types of stochastic control laws to stabilize the underlying system in various senses.

- The results discussed in this paper show clearly that that the stability theory for SDEs is much richer than that for ODEs. 


\section{References}

[1] Arnold, L., Stochastic Differential Equations: Theory and Applications, John Wiley Sons, 1972.

[2] L. Arnold., Stabilization by noise revisited. Zeitschrift fur Angewandte Mathematik und Mechanik 70 (1990), 235-246.

[3] L. Arnold, H. Crauel and V. Wihstutz, Stabilization of linear systems by noise, SIAM J. Control and Optimization 21(1983), $451-461$.

[4] Caraballo, T., Liu, K. and Mao, X., On stabilization of partial differential equations by noise, Nagoya Mathematical Journal 161(2001), 155-170.

[5] Friedman, A., Stochastic Differential Equations and Their Applications, Academic Press,, 1976.

[6] Has'minskii, R.Z., Stochastic Stability of Differential Equations, Sijthoff and Noordhoff , 1981.

[7] Kushner, H.J., Stochastic Stability and Control;, Academic Press, 1967.

[8] Ladde, G.S. and Lakshmikantham, V., Random Differential Inequalities, Academic Press, 1980. 
[9] Mao, X., Stability of Stochastic Differential Equations with Respect to Semimartingales, Longman Scientific and Technical, 1991.

[10] Mao, X., Exponential Stability of Stochastic Differential Equations, Marcel Dekker, 1994.

[11] Mao, X., Stochastic stabilization and destabilization, Systems and Control Letters 23(1994), 279-290.

[12] Mao, X., Stochastic self-stabilization. Stochastics and Stochastics Reports, 57(1996), 57-70.

[13] Mao, X., Stochastic Differential Equations and Applications, Horwood, 1997.

[14] Mao, X., Stochastic versions of the LaSalle theorem, J. Differential Equations 153(1999), 175-195.

[15] Mao, X., Some contributions to stochastic asymptotic stability and boundedness via multiple Lyapunov functions, J. Math. Anal. Appl. 260(2001), 325-340.

[16] Scheutzow, M., Stabilization and destabilization by noise in the plane. Sto. Anal. Appl. 11(1993), 97-113. 